\title{
Application of Piezoelectric Materials on Repair of Delaminated Structures
}

\author{
Quan Wang* \\ Department of Mechanical and Manufacturing Engineering, University of Manitoba, Winnipeg, MB, Canada
}

\begin{abstract}
The potential of piezoelectric ceramics on repair of delaminated structures subjected to a static loading is researched in the letter. A sliding fracture mode on two tips of a delaminated beam structure was uncovered by the author in a previous report. The stress singularity due to the fracture mode at the tips is attempted to be mended with a pair of piezoelectric patches by virtue of their electro-mechanical characteristics. A developed mechanics model is employed to study the applicability of the piezoelectric materials in repairing four types of delaminated structures, i.e. simply supported, cantilevered, propped cantilevered, and fixed beams. The effectiveness of the proposed method is particularly investigated with respect to the locations of the delamination in both the thickness and longitudinal directions of the beams. It is hoped that the research findings in the letter will promote an application of smart materials to repair delaminated or cracked engineering structures.
\end{abstract}

\section{INTRODUCTION}

Damage in aerospace, aeronautical, mechanical, civil and offshore structures often results from reasons such as fatigue, corrosion, or accidents. Such damage when left unattended can grow at alarming rate due to the singularity of the stress and strain in the vicinity of the damage. This can lead to an increase in vibration level, reduction in load carrying capacity, deterioration in the normal performance of the component, and even catastrophic failure [1-3]. Delamination in composites has been a topic of concern and this has been extensively reviewed [4]. Effective repair of structural damage is therefore an important and practical topic. Repair can extend the service life and can be a cost efficient alternative to immediate replacement of the damage component. Conventional repair methods usually involve welding, riveting or mounting additional patches to the parent structure without removing the damaged portion and their limitations have been reviewed by Baker et al. [5]. This review highlighted the need for more effective repair methods to be developed as the strains and stresses in the areas surrounding the damaged domain may cause additional damage that severe reduction in the service life of the entire structure maybe the result if left unattended. In addition, welding or bonding patches can cause significant stress alteration and serious stresscorrosion problems and increase the burden on weight sensitive structures [6-7]. The limitations of conventional repair methods imply the need to look for more effective ways to repair damage.

The advent of smart materials such as piezoelectrics, and shape memory alloys opens up new opportunities for improved repair techniques to overcome some of the limitations of conventional repair methods. For example, embedded shape memory alloy in structures will not significantly affect the strain and stress in areas surrounding the damage. Both piezoelectric materials and shape memory alloys enable repair adjustments to external loading changes, as indicated in a research by Wang et al. [8] on repair of a surface cracked

*Address correspondence to this author at the Department of Mechanical and Manufacturing Engineering, University of Manitoba, Winnipeg, MB, Canada; E-Mail: Q_Wang@Umanitoba.ca beam subjected to static loading via piezoelectric patches. Wang and Quek [9] developed a simple mechanics model for repair of delaminated beams subjected to concentrated static loading via piezoelectric patches. Their analysis exposed that sliding mode fracture is induced at the tips of the delamination, leading to stress singularity. A simple repair methodology via piezoelectric patches was attempted to remove this singularity. However, a slight opening fracture mode may be stimulated from the model which was found recently by the author's group. Duan et al. [10] proposed a finite element analysis on repair of a delaminated simply supported beam structure via piezoelectric materials. Results on the effect of the delamination locations, external loading locations, and the length of piezoelectric patches on the effectiveness of the piezoelectric materials were summarized for the specific delaminated structure with pin ends.

The objective of this paper is to apply piezoelectric patches in repairing general delaminated beams with four types of boundary conditions, i.e. simply supported, cantilevered, propped cantilevered, and fixed ends. The effectiveness of the piezoelectric patches on the delaminated structures versus the relative locations of the delamination to the external loading and the boundaries of the beam were particularly investigated, and conclusions on the effectiveness are summarized.

\section{MECHANICS MODEL FOR REPAIR OF DELAMI- NATED BEAMS WITH PIEZOELECTRIC PATCHES}

A general delaminated beam shown in Fig. (1a) subjected to a static loading $P$ is to be repaired using piezoelectric patches. The material and geometric parameters of the delaminated beam are denoted as $E$ for the Young's modulus of the host beam, $T$ its width, $H$ its thickness, $t$ the thickness of the upper layer of the delamination and $a$ the length of the delamination. Two piezoelectric patches with the same thickness $h_{p}$, and length $L_{p}$ are attached on the upper and lower surfaces of the beam, respectively. The delamination tip is located at the center of piezoelectric patches such that a uniform moment induced by the piezoelectric layer can be induced on the tip section. The voltages applied on the top and bottom piezoelectric patches are denoted as $V_{1}$ and $V_{2}$ for the repair purpose. 


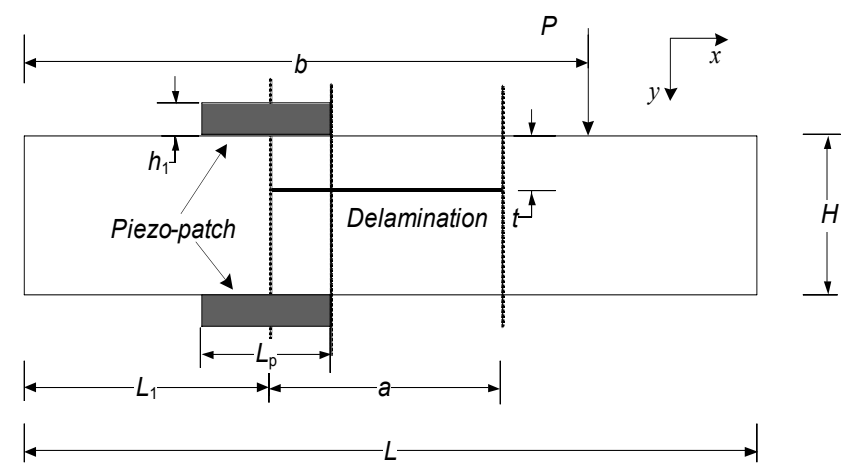

Fig. (1a). A delaminated beam structure subjected to an external loading and attached with a pair of piezoelectric ceramics.
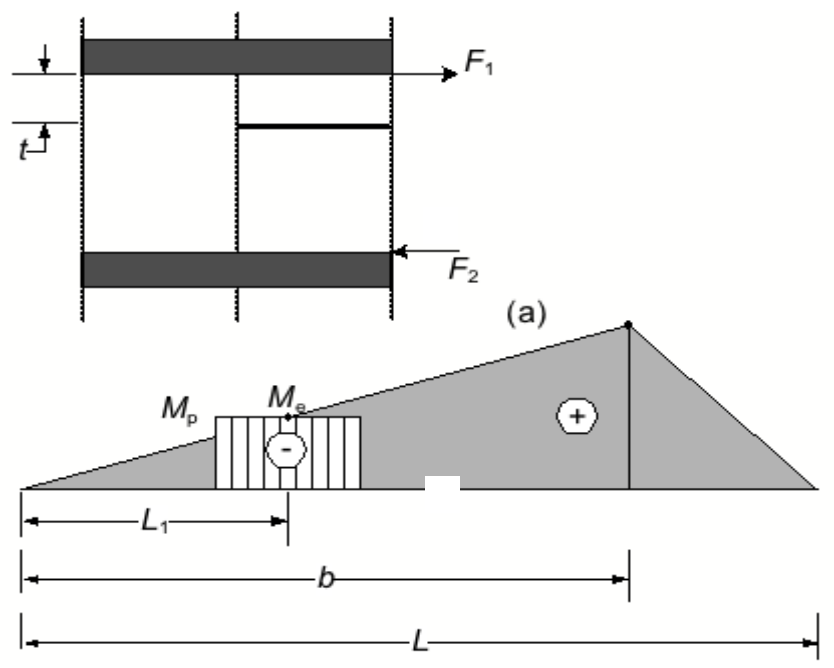

(b)

Fig. (1b). Force induced on the interface of the piezoelectric layer and the substrate beam and the moment diagram of the delaminated beam.

Based on the analysis of the delaminated beam by Wang and Quek [9], the sliding fracture mode is induced by the moment at two tip locations due to the external static loading. A simple quantitative analysis was provided in reference [9]. On the other hand, it can also be understood that the compression and tension with same magnitude on the upper and lower layers of the delamination are clearly present under the external force, based on classical beam theory, which definitely lead to the sliding fracture mode on the tips of the delamination. Such fracture mode will give rise to stress singularity at the tips. Thus, the key in the design is to employ the electromechanical characteristics of piezoelectric materials to wipe out the effect of the moment by the external loading at the locations of the two tips, and accordingly erase the sliding fracture mode. The moments induced by external force $P$ and shear forces $F_{1}$ and $F_{2}$, induced at the interface of the piezoelectric ceramics and the substrate beam structures, are plotted in Fig. (1b). The moment at the left tip of the delamination, for example, induced by external load is expressed by $M_{e}\left(P, b, L_{1}\right)$ as a function of the external force, its location, and the delamination location. From the diagram in Fig. (1b), the uniform moment by the pair of piezoelectric ceramics is approximately written as follows according to mechanics of material principal:
$M_{p}=F_{1} t+F_{2}(H-t)$

In classical beam theory, the possible tension or compression on an element in the structure is purely due to the existence of moment induced in the structure and proportional to the moment. Therefore, the upper and lower layers of the delamination will be in a neutral state if the expression

$M_{p}+M_{e}=0$

is set, or if the uniform moment induced by the piezoelectric patches can balance the effect of the external force. It has to be noted according to mechanics of materials that the shear forces $F_{1}$ and $F_{2}$ will also independently bring flexural deformations on the upper and lower layers of the delimitation at the location of the right tips of the piezoelectric layers. In order not to bring additional opening fracture mode on the tip of the delamination, the induced flexural deformations need to be with same magnitude. The upper and lower layers of the delamination at the domain of the interaction of the piezoelectric layers and the substrate can be simply modeled as two cantilevers subjected to moments of $F_{1}$ and $F_{2}(H-t)$, respectively. Therefore, the expressions for the flexural deformation are simply expressed as: $F_{1} t L^{2} / 2 E I_{1}$ and $F_{2}(H-t) L^{2} / 2 E I_{2}$, where $I_{1}$ and $I_{2}$ are moment of inertia of the cross section of the two layers. Hence, the condition for the equal deformation discussed above is simply expressed as,

$\frac{F_{1}}{t^{2}}=\frac{F_{2}}{(H-t)^{2}}$

In view of Eqs. (1-3), the force induced at the interfaces of the piezoelectric patches and the substrate, $F_{1}$ and $F_{2}$, should be given as:

$$
\begin{aligned}
& F_{1}=\frac{M_{e} t^{2}}{(H-t)^{3}+t^{3}} \\
& F_{2}=\frac{M_{e}(H-t)^{2}}{(H-t)^{3}+t^{3}}
\end{aligned}
$$

Once the shear forces can be induced by the piezoelectric layers according to Eqs. (4a-b), neither the opening nor the sliding fracture modes can exist at the tip of the delamination. Hence the repair of the delaminated beam can be achieved from the simple mechanics model. It has to be noted that the effect of the moment distribution is not modeled in the letter, especially for indeterminate beam structures. Such effect is assumed to be small as the reaction forces on the boundaries are almost independent on the characteristics of the delamination if it is not long. More investigations on a proper model are necessary when the delamination is long enough to have effect on the moment distribution in indeterminate beams. In reference [9], same shear forces were proposed in the design of the piezoelectric layers, which can only ease the sliding fracture mode but will induce additional opening mode at the tip. Crawley and de Luis [11] proposed the following expression for a shear force between the substrate and the piezoelectric patch shown in Fig. (1b) as a function of the voltage applied on the piezoelectric layer:

$F=\frac{E H T}{\psi+6} \Lambda$ 
where $\psi=E H / E_{p} h_{p} ; \Lambda=d_{31} V / h_{p} ; E_{p}$ the equivalent Young's modulus of the piezoelectric layer for one-dimensional problem; $T$ the depth dimension of the piezoelectric layer and the substrate; and $d_{31}$ the piezoelectric charge coefficient. It was also indicated that the force transferred between the piezoelectric layer and the substructure is over an infinitesimal distance near the ends of the actuator if an infinitely stiff bonding is provided. From the relationship between the induced shear force and the applied voltage shown in Eq. (5), the designed voltages on the upper and lower piezoelectric patches are thus determined as:

$V_{1}=\frac{h_{p}(\psi+6) t^{2}}{d_{31} E H\left((H-t)^{3}+t^{3}\right)} \frac{M_{e}}{T}$

$V_{2}=\frac{h_{p}(\psi+6)(H-t)^{2}}{d_{31} E H\left((H-t)^{3}+t^{3}\right)} \frac{M_{e}}{T}$

In a recent research by the author's group, the design method was verified via finite element analysis (FEM) on a delaminated simply supported beam. The difference of the designed voltages between Eqs. (6a-b) and the voltages used in FEM is within an acceptable percentage, i.e. less than $8 \%$ when the external force is outside the delamination domain. Next, the proposed design is to be applied to four types of delaminated beams.

\section{REPAIR OF DELAMINATED BEAM WITH PIEZOE- LECTRIC PATCHES: NUMERICAL SIMULATIONS AND DISCUSSIONS}

The geometrical and material properties of the delaminated beams and the piezoelectric patches are shown in Table 1. The external static force in all the following simulations is set to be $P / T=10000 \mathrm{~N} / \mathrm{m}$. Such force will induce a deformation about $1.5 \mathrm{~mm}$ at the free end of a cantilever with the properties in Table 1. As concluded by reference [10], the length of the piezoelectric patches has less effect on the repair results, the length of the piezoelectric patches are thus set to be $0.1 \mathrm{~m}$. For each of the piezoelectric pair, the voltage at the upper and lower piezoelectric layers should be applied with different alignment of the poling direction of the piezoelectric patch such that tension and compression may be induced accordingly.

Table 1. Geometrical and Mechanical Property of the Delaminated Beams and the Piezoelectric Layers

\begin{tabular}{|c|c|c|c|}
\hline \multicolumn{2}{|c|}{ Substrate (Steel) } & \multicolumn{2}{c|}{ Piezoelectric Ceramics } \\
\hline \hline$L(\mathrm{~m})$ & 1 & $L_{p}(\mathrm{~m})$ & 0.1 \\
\hline$E\left(N / m^{2}\right)$ & $210 \times 10^{9}$ & $E_{\mathrm{p}}\left(N / \mathrm{m}^{2}\right)$ & $63 \times 10^{9}$ \\
\hline \multirow{2}{*}{$H(\mathrm{~m})$} & \multirow{2}{*}{0.05} & $H_{p}(\mathrm{~m})$ & 0.005 \\
\cline { 3 - 4 } & & $d_{31}(\mathrm{~m} / \mathrm{V})$ & $190 \times 10^{-12}$ \\
\hline
\end{tabular}

First, a delaminated simply supported beam is investigated. In Fig. (2a), the voltages on the upper piezoelectric layers on the left and right tips of the delamination are plotted with respect to the location of the center of the delamination given the external loading at the middle of the beam. In the simulation, the delamination is placed at the mid of the thickness direction, thus the voltages on the lower payers are with same amplitude but with opposite sign. It is seen that no voltages are necessary when the tips of the delamination are on the boundaries of the beam. The findings are simply due to the fact that the two boundaries of the beam are free of rotation, thus no any moment could be induced at the end, and hence no repair is needed. Another observation from the figure is that when the delamination moves to the loading position, higher voltages are simulated indicating more effort is needed for the repair. The highest voltage is about $5000 \mathrm{~V}$ when the delamination is at the mid of the beam since the moment induced by the external force reaches maxima at the loading position. The voltage on the left patch is smaller at first when the delamination is at the left of the loading, and will become larger than the right patch when the delamination is at the right side of the loading.

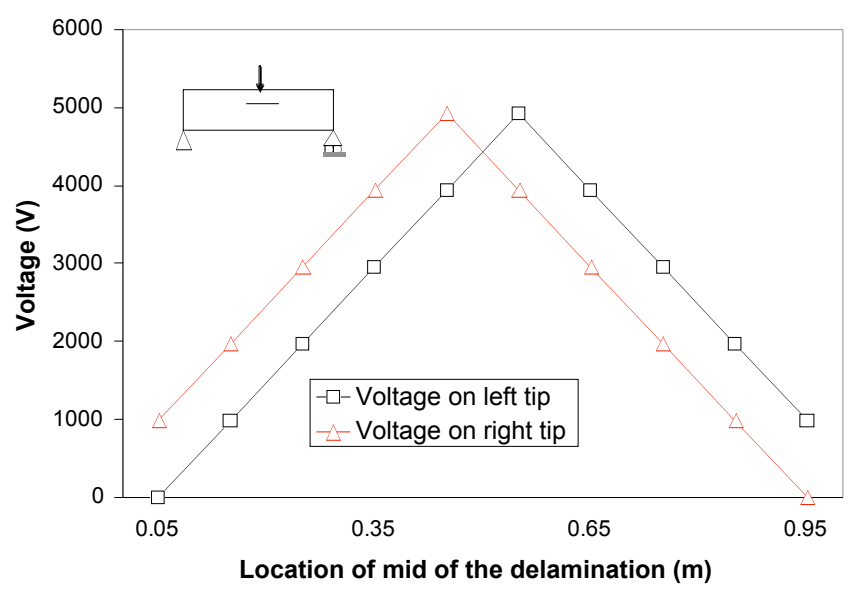

Fig. (2a). Repair of a delaminated simply supported beam.

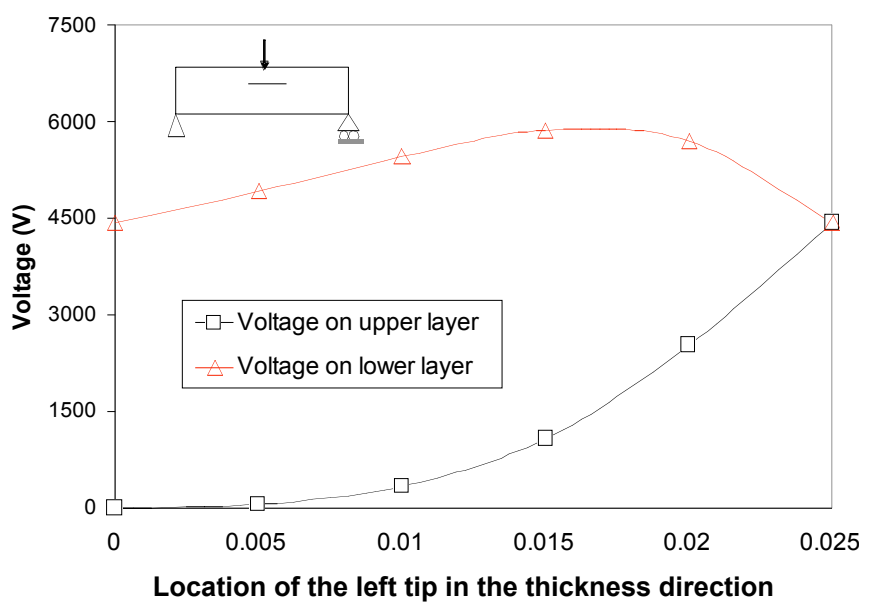

Fig. (2b). Voltages with respect to the location of the delamination in the thickness direction of the simply supported beam.

To investigate the variation of the designed voltages with respect to the location of the delamination in the thickness direction, the voltages on the upper and lower piezoelectric patches on the left tip of the delamination located at the mid of the longitudinal direction of the beam are illustrated in Fig. (2b). It is observed that the closer the delamination is to the upper surface of the beam, the lower voltage necessities for the upper piezoelectric patch. It is obvious to find no voltage is necessary when the delamination is at the surface, or no delamination exists at all. On the other hand, the ap- 
plied voltage on the lower patch increases to the highest value when the delamination reaches about one third of the thickness direction, and after that the voltage decreases and converges to a value about $4500 \mathrm{~V}$ for both the patches when the delamination is at the middle. Different voltages on the piezoelectric patches are used to avoid any possible opening fracture mode as discussed before. Since the key in the design of repair is to induce moment via the piezoelectric patches to balance the moment at the tips of the delamination by the external loading, a non-zero voltage on the lower patch is still necessary for the beam even when the delamination is at the top surface and no repair is unnecessary from common sense. Although the observation in Fig. (2b) is from the simulation on a simply supported beam, it is universally valid for other types of delaminated beam, and hence will not be investigated in the following studies in which only the locations of the delamination in the longitudinal direction of the delaminated beam are to be studied.

Second, the variation of the voltages on the upper two piezoelectric layers with respect to the delamination location for a delaminated cantilevered beam subjected to a static loading at its free end is shown in Fig. (3). It is clearly seen that higher voltages are required when the delamination is close to the fixed end of the beam, which is with highest moment and extremely high voltage needed. Thus, it is practically impossible for repair if the delamination is close to the fixed end. It can also be imagined that no repair is desired at all when the delamination is at the loading position or at the right side of the loading when the force is not at the free end of the beam. The voltage on the left piezoelectric patch is always bigger than that on the right patch in the simulations.

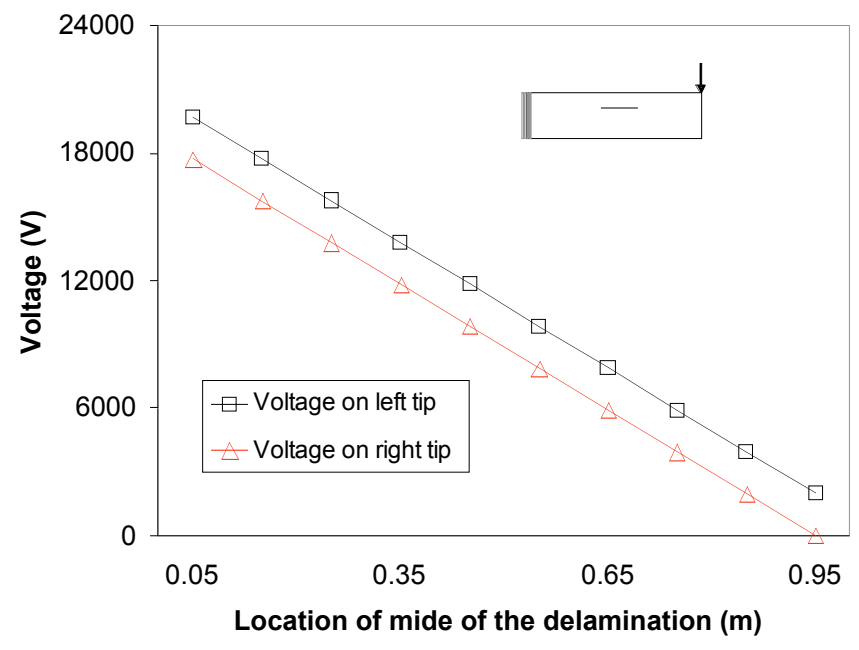

Fig. (3). Repair of a delaminated cantilevered beam.

Finally, studies on a delaminated propped cantilevered beam and a fixed beam are conducted in Figs. (4,5). In both beams, external loading is subjected at the mid of the beams. In the simulations, the delamination effect on the moment distribution in the structure is neglected to simplify the calculation since the effect is not influential in calculating reactions on the boundaries although it is dominant for the stress distribution around the tips of the delamination. Some interesting results are observed from the two figures. It is natural to find that the lower voltage is necessary if the delamination is close to the pin end in the propped cantilevered beam. In the two beams, different from the simply supported and cantilevered beam, higher voltage is required on the left patch than that on the right patch when the patches are compressed, while lower voltage is necessary on the left patch when tension is excited on the two patches after the delamination moves after one third of the length of the beam from the left end of the beams. However, the voltage on the left patch dominates again when patches are at the right side of the loading position in both cases if the patches are in tension. Another interesting observation is that the magnitude of the voltage on the left patch is lower when it is at the loading position rather than the fixed position for the propped cantilevered beam, whereas the magnitude of the voltage on the right patch is lower when it is at the fixed end. The observation is not universal and only applies to the scenario when the loading is at the mid of the beam. When the loading is at any other location, further simulations are required to find conclusion on the voltages on the two patches. The complexity of the variation of the voltages is due to the complicated moment distributions in the indeterminate beam structures and the length effect of the delamination. Nevertheless, it is under expectation that the larger the length of the delamination, the higher difference of the voltages on the two patches in all the four types of the delaminated beams. It can also be imagined that the thinner the substrate beam, the lower the voltages on the piezoelectric patches as also pointed by the reference [9]. In all the simulations, the voltages on the piezoelectric patches are lower than their allowable voltage intensity, i.e. $1 \sim 2 \mathrm{kV} / \mathrm{mm}$.

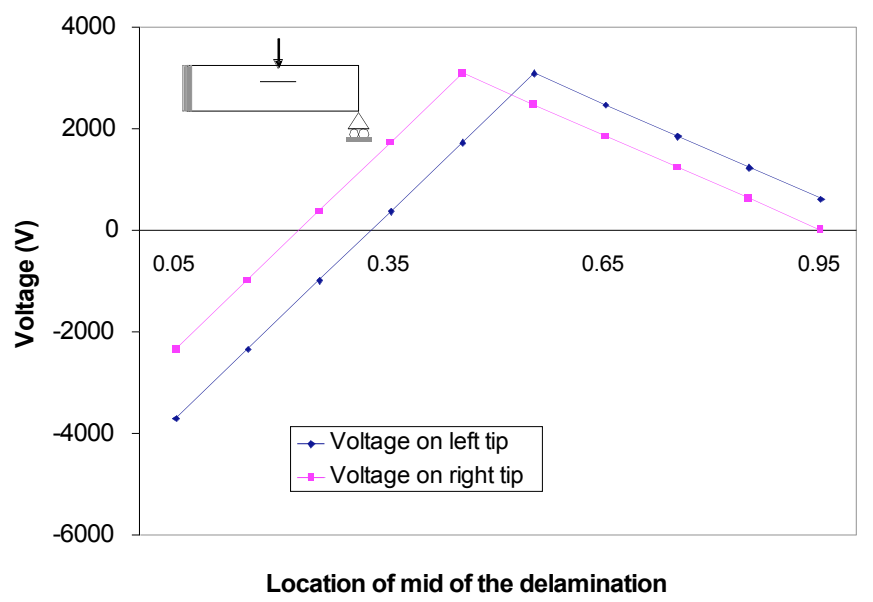

Fig. (4). Repair of a delaminated propped cantilevered beam.

\section{CONCLUSIONS}

An effective repair design is proposed in the letter to lessen the stress singularity at the tips of the delamination due to the sliding fracture by the external loading, while avoid the opening fracture mode induced by the equal voltage design. The effective repair design is applied to four more types of delaminated beams with different boundary conditions in the letter. The simulation results show that higher voltages are necessary when the delamination is close to the fixed end in all beams with fixed ends. In addition, it is found that higher voltages are required when the 


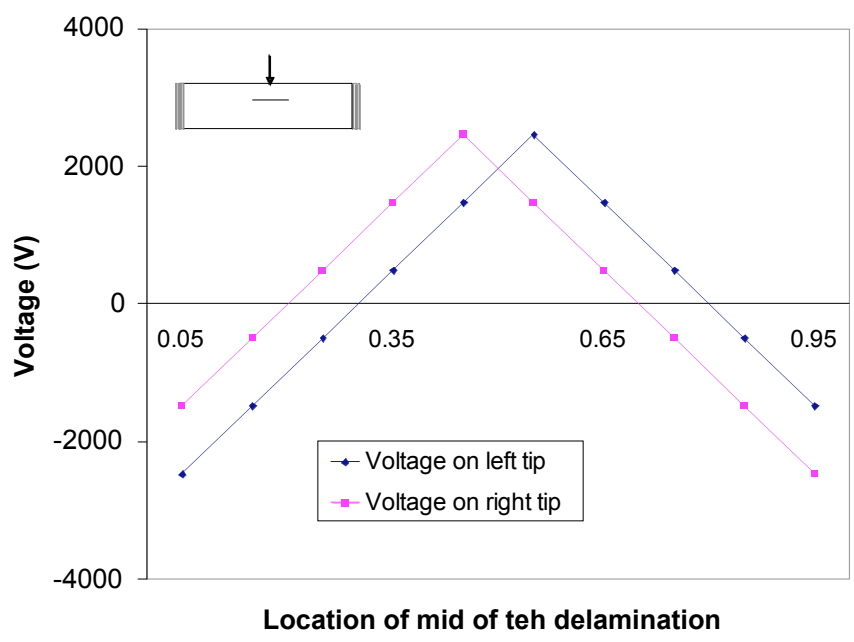

Fig. (5). Repair of a delaminated fixed beam.

delamination is close to the loading position for simply supported, propped cantilevered, and fixed beam, but opposite conclusion is found in cantilevered beam. Comparison of the voltages on the left and right piezoelectric patch is simple in the simply supported and cantilevered beams, but seems complicated due to the moment distribution in the indeterminate propped cantilevered and fixed and the length of the delamination when the loading is at other locations rather than the mid of the beams. The variation of the voltages on the upper and lower piezoelectric patches at a certain tip of the delamination is also studied with respect to the location of the delamination in the thickness direction. Further studies will include the effectiveness of the repair design for delaminated or cracked beams with complicated external loading including dynamic loadings. In addition, the application of other smart materials, such as shape memory alloys, on repair of the damaged structures needs further investigation as the ability of the piezoelectric ceramics is limited in inducing larger forces on the substrate, and thus restricts their applicability in repairing stiffer engineering structures. It is noted from simulations that the variations of the voltages on the piezoelectric patches for the four types of beam structures do follow the moment distributions for the corresponding structures. However, it has to be pointed out that a more accurate derivation of the variations of the voltages for the indeterminate propped cantilevered and fixed beam structures should take into account the effect of the delamination since the effect of the delamination is assumed to be negligible to the moment distribution in the current research. In addition, the piezoelectric coupled effect is not modeled in the simulations. The motive of the research is to show the potential of the piezoelectric materials in repairing delaminated structures and the effect of the location of the delamination on the design. Smart materials enable researchers to look beyond the available repair methods to more active repair methods that will be responsive to external condition changes. This is because their strength and their interaction with the repaired structure can be adjusted with environmental changes due to their smart characteristics, and because they cause less stress concentration on the repaired structures. It is hoped that the research in the letter can promote the applications of smart materials in repair of engineering structures.

\section{ACKNOWLEDGEMENT}

The author acknowledges the support from the Canada Research Chair (CRC) program by the Canadian government and the University of Manitoba.

\section{REFERENCES}

[1] H. H. Yu, M. Y. He, J. W. Hutchinson, "Edge effects in thin film delamination", Acta Mater., Vol. 49, pp. 93-107, 2001.

[2] G. N. Wells, R. de Borst, and L. J. Sluys, "A consistent geometrically non-linear approach for delamination", Int. J. Numer. Methods Eng., Vol. 54, pp. 1333-55, 2002.

[3] N. A. Chrysochoidis and D. A. Saravanos, "Assessing the effects of delamination on the damped dynamic response of composite beams with piezoelectric actuators and sensors", Smart Mater. Struct., Vol. 13, pp. 733-42, 2004.

[4] D. J. Wilkins, J. R. Eisenmann, R. A. Camin, W. S. Margolis, and R. A. Benson, "Characterizing delamination growth in graphiteepoxy Damages in Composite Materials", ASTM Special Technical Publication, pp. 168-83, 1982.

[5] A. A Baker, L. R. F. Rose, and R. Jones, Advances in the bonded composite repair of metallic aircraft structure. Boston: Elsevier, 2002 .

[6] L. J. Hart-Smith, "Adhesive mount stresses and strains at discontinuities and cracks in mounted structure", J. Eng. Mat. Technol., Vol. 100, pp. 16-24, 1978.

[7] A. A. Baker and R. Jones, Bonded repair of aircraft structure. The Netherland: Martinus-Nijhoff Publishers, 1988.

[8] Q. Wang, S. T. Quek, and K. M. Liew, "On the repair of a cracked beam with a piezoelectric patch', Smart Mater. Struct., Vol. 11, pp. 404-410, 2002

[9] Q. Wang and S. T. Quek, "Repair of delaminated beams via piezoelectric patches", Smart Mater. Struct., Vol. 13, pp. 1222-9, 2004

[10] W. H. Duan, S. T. Quek, and Q. Wang, "Finite element modeling on repair of delaminated beam via piezoelectric patches", Smart Mater. Struct., submission.

[11] E. F. Crawley and J. de Luis, "Use of piezoelectric actuators as elements of intelligent structures", AIAA J., Vol. 25 pp. 1373-85, 1987. 